\title{
Estrategias y desafíos en extensión universitaria: la experiencia del Observatorio de Conflictos Sociales del Nordeste Argentino
}

\section{Strategies and challenges in university extension: The experience of the Observatorio de Conflictos Sociales del Nordeste Argentino}

\author{
Observatorio de Conflictos Sociales Nordeste ${ }^{1}$ \\ Recibido: 16/10/2020. Aceptado: 16/04/2021 \\ http://dx.doi.org/10.30972/dpd.10154827
}

\begin{abstract}
Resumen
El artículo tiene como finalidad presentar una reflexión colectiva sobre las acciones y estrategias que, a lo largo del tiempo, fueron configurando y modificando el modus operandi del Observatorio de Conflictos Sociales del Nordeste Argentino. El principal objetivo de este Observatorio es el de realizar registros y análisis de la conflictividad social regional en sus dimensiones ambientales, de género y de Derechos Humanos, en interacción con las organizaciones sociales e instituciones que abordan las problemáticas correspondientes. Por lo tanto, se tematizan también esas vinculaciones y los recorridos por cada una de las tres áreas de trabajo.
\end{abstract}

Palabras clave: estrategias; conflicto social; extensión universitaria.

1 Participantes: Fernando Nahuel Marturet, integrante del Observatorio de Conflictos Sociales del NEA y profesor en Filosofía (Unne), correo electrónico: fernandomarturet@gmail.com; Marisa Noemí Miño, integrante del Observatorio de Conflictos Sociales del NEA y profesora en Filosofía (Unne), correo electrónico: mmnoemi9@gmail.com; Matías Lihuel Cendali Godoy, integrante del Observatorio de Conflictos Sociales del NEA, estudiante del Profesorado y la Licenciatura en Filosofía (Unne), correo electrónico: lihuel.cg@live.com; Maximiliano Eduardo Román, integrante del Observatorio de Conflictos Sociales del NEA, profesor en Filosofía (Unne) y profesor adjunto a cargo en las cátedras Introducción a la Problemática Filosófica, Problemática Filosófica Contemporánea y Filosofía Argentina y Latinoamericana (Unne), correo electrónico: maxiroman@hotmail.com; Nicolás Silva, estudiante de la Licenciatura en Comunicación Social (Unne), correo electrónico: 1nicosilva@gmail.com; Raquel Adriana Carmona, integrante del Observatorio de Conflictos Sociales del NEA y licenciada en Relaciones Internacionales (UNR), correo electrónico: racarmona.1@gmail.com; Sofía Raquel Cardozo Quintana, integrante del Observatorio de Conflictos Sociales del NEA y estudiante del Profesorado y la Licenciatura en Filosofía (Unne), correo electrónico: soficardozoq@gmail.com; Ana Clara Black, asesora técnica de la Red de Salud Popular Dr. Ramón Carrillo, organización no gubernamental, correo electrónico: reddesaludchaco@yahoo.com.ar; María Alejandra Gómez, coordinadora de la Red de Salud Popular Dr. Ramón Carrillo, organización no gubernamental, correo electrónico: reddesaludchaco@yahoo.com.ar

Dossier "Metodologías participativas alternativas o contrahegemónicas: otros 


\begin{abstract}
This article aims to present a collective reflection about the actions and strategies that, throughout the time, have been configuring and modifying the modus operandi of the Observatorio de Conflictos Sociales del Nordeste Argentino. The principal objective of this observatory is to register and analyze the social contentiousness of the region in their environmental, about genders and human rights dimensions, in interaction with the social organizations and institutions that approach the corresponding problematics. Therefore, it is also considered those entailments and their trajectory in each of the areas of work.
\end{abstract}

Keywords: strategies, social contentiousness, university extension.

\title{
Introducción
}

El Observatorio de Conflictos Sociales del Nordeste Argentino (OCSo-NEA) surge en 2017 como iniciativa de un grupo de docentes, investigadores y estudiantes de la Universidad Nacional del Nordeste, con el objetivo de ampliar las prácticas de investigación, extensión y producción de conocimientos a partir de las articulaciones previamente existentes con organizaciones sociales de la región. Su concreción fue posible, en parte, por el financiamiento obtenido durante tres períodos consecutivos en las convocatorias del programa Universidad, Cultura y Sociedad, de la Secretaría de Políticas Universitarias del Ministerio de Educación de la Nación. Sus antecedentes directos son la creación del Grupo de Investigación sobre Conflictos Sociales (GrICSo) y las jornadas interdisciplinarias sobre conflictos y problemáticas sociales en la región del Gran Chaco (realizadas bianualmente desde 2011).

Nuestro marco teórico y metodológico inicial sostiene que los conflictos constituyen un elemento fundamental para la comprensión de la dinámica social, en tanto emergente de un trasfondo de permanente lucha de clases. Un registro sistemático, sincrónico y permanente de los conflictos sociales en el Nordeste de la Argentina y el análisis de sus características pueden constituir el punto de partida para la elaboración de políticas públicas y de estrategias colectivas tendientes a solucionar las causales de los conflictos detectados y/o mitigar su impacto sobre las poblaciones vulneradas. Este registro es sistemático, ya que se determinan las unidades de análisis específicas de los conflictos que se analizan: feminicidios, ataques de odio, denuncias por fumigaciones con agrotóxicos, muertes en manos del aparato represivo del Estado, represión policial y hechos de protesta social, a la vez que se establecen las fuentes de los datos a relevar: 
medios periodísticos, archivos de las organizaciones con las que trabajamos, fuentes judiciales, encuestas, entrevistas, entre otras. Esto permite atender los aspectos mensurables de los fenómenos estudiados que dan cuenta de cierta "temperatura" de la conflictividad, por eso, además, es necesario abarcar la temporalidad y coyuntura de los conflictos de forma sincrónica y permanente.

En este artículo, que recorre la trayectoria realizada por el Observatorio, intentaremos detallar las distintas estrategias y acciones que sus integrantes hemos realizado para producir conocimiento junto con y para las organizaciones sociales desde una perspectiva crítica y comprometida con los Derechos Humanos. En paralelo, proponemos reflexionar sobre los desafíos que las tareas de extensión universitaria significan para cada área en particular y para el equipo en general.

En primera instancia, se describe a modo general la metodología básica que utilizamos en el OCSo-NEA y posteriormente, las memorias de cada una de sus áreas: Derechos Humanos, género y diversidad, ambiente y territorio. Finalmente, una conclusión acerca del camino recorrido hasta la fecha, cuyo final permanece abierto.

\section{Formación, registro y difusión: una metodología general}

Las primeras elaboraciones del OCSo-NEA surgieron en torno a la creación de matrices de datos sobre hechos de protesta en cada provincia del Nordeste Argentino (Chaco, Corrientes, Formosa y Misiones). El objetivo central de ese trabajo era elaborar un mapeo general, sistemático y permanente de la conflictividad social en la región (Pratesi, Graciosi y Román, 2018). No obstante, el gran volumen de información relevado significó un obstáculo para lograr avances significativos teniendo en cuenta la cantidad de personas que integramos el equipo (un grupo estable de 4 personas que fue cambiando y se amplió a 6) y la novedad que significaba el tipo de tarea abordada (a diferencia de las investigaciones estrictamente académicas que veníamos realizando). Por lo tanto, decidimos colectivamente posponer el trabajo sobre hechos de protesta hasta que estuvieran dadas las condiciones para materializarlo plenamente. A partir de indagaciones previas de algunes integrantes, decidimos entonces avanzar en investigaciones más acotadas en lo temático, en lo temporal y en lo espacial, a partir de la división en áreas de trabajo manteniendo un mismo marco teórico-metodológico.

Así fue como en 2017 comenzó a trabajar un área específica de género y diversidad y en 2018 consolidamos el trabajo en tres áreas: género y diversidad, ambiente y territorio, y Derechos Humanos. Cada área elaboró una propuesta de trabajo a partir de sus vinculaciones con organizaciones sociales que abordaban la temática. Actualmente, el Observatorio ha logrado 
consolidar un equipo de trabajo compuesto por 6 integrantes, en su mayoría estudiantes, docentes e investigadores provenientes del campo de la filosofía. Naturalmente, esta base de conocimientos, si bien otorga medios para interpretar y problematizar la conflictividad social, no son suficientes para llevar adelante las investigaciones empíricas que estos fenómenos requieren para ser conocidos. Aunque esto supone siempre una gran limitación, como equipo nos proveímos de una constante formación, por medio de cursos, seminarios y mesas de discusión científica a las que nos hemos presentado, lo que nos ha permitido hacernos de otros saberes y perfeccionar metodologías de registro y análisis de datos.

A nivel general, la metodología que utilizamos desde el OCSo-NEA se organiza en tres grandes etapas: formación, registro y difusión.

Las reuniones organizativas y talleres de formación teórico-metodológica fueron realizados en los inicios del grupo con el propio equipo extensionista, entre quienes ya conforman el espacio y quienes pudieron haberse incorporado recientemente. En paralelo, iniciamos las reuniones con las organizaciones de la sociedad civil coparticipantes, en las cuales se sientan las bases del trabajo a realizar, se toman decisiones en conjunto para coordinar tareas y se relevan necesidades de registro, sistematización y análisis de datos. Estas instancias se repiten periódicamente de acuerdo con las necesidades de formación (generales o sobre algún tema/técnica específicos) o de acuerdo con el momento de desarrollo de la investigación y sus avances.

En una segunda etapa, preparamos los registros, diseñando los instrumentos y procedimientos para lograr que los mismos sean fiables y homologables. Luego de consultar su viabilidad con las organizaciones coparticipantes, procedemos a la recolección de información de las distintas fuentes (escritas/orales, directas/indirectas, primarias/secundarias). Al concluir el período, procedemos al análisis, mediante el procesamiento de la información recolectada a nivel provincial, en primera instancia, y a nivel regional, en segundo término, a través de reuniones por área y generales.

En la tercera etapa de difusión, se organizan instancias de divulgación de resultados parciales, bajo diversos formatos y en diferentes ámbitos, que permitan una retroalimentación tanto con investigadores académicos como con las organizaciones coparticipantes. En segundo lugar, procedemos al diseño de publicaciones acordes a las necesidades de ambos destinatarios principales $y$, finalmente, se presentan las mismas a través de formatos académicos (papers, reuniones científicas) y no académicos (encuentros o talleres con las organizaciones coparticipantes, medios de comunicación, redes sociales). 


\section{Memorias del área Derechos Humanos}

En 2018, el área de Derechos Humanos comenzó a trabajar sobre la base de investigaciones previas de integrantes del equipo, la experiencia de militancia antirrepresiva y la relación con la Red Corrientes de Derechos Humanos (RCDH). Esta organización, conformada de hecho hace más de diez años, cuenta con una sólida trayectoria en la promoción participativa de los derechos de las personas en condición de vulnerabilidad, el acompañamiento y la intervención ante derechos conculcados o amenazados. Asimismo, promueve la lucha por el esclarecimiento, la justicia y contra la impunidad de crímenes cometidos por el Estado, delitos de lesa humanidad y violencia represiva, tanto en dictadura como en el marco de la institucionalidad democrática. Es la única organización de la sociedad civil correntina que realiza autogestivamente actividades educativas, artísticas y de promoción de derechos dentro de las unidades penales.

Durante las primeras reuniones entre les integrantes del OCSo-NEA y la RCDH se definió la temática a analizar y se detalló la metodología de trabajo. El primer informe realizado fue Muertes por el aparato represivo del Estado en la provincia de Corrientes (2008-2018). Su construcción -y la elaboración de la base de datos de la que se desprende- demandó prácticamente un año de trabajo, entre conversaciones preliminares, definición y redefinición de parámetros, variables y enfoque. La fuente principal de información fueron los listados previamente elaborados por la RCDH, cuya sistematización y normalización quedó a cargo del OCSo-NEA. Cabe señalar que, si bien el informe recorta los registros vinculados a una década, el insumo original contenía un total de 128 casos de personas asesinadas por responsabilidad del Estado correntino desde el retorno de la democracia (1983).

Una versión preliminar de ese informe fue presentada el 18 de diciembre de 2018 en el Centro Cultural Universitario de la Unne. Su motivación principal fue la muerte de María Elena Frette, una interna del Penal de Mujeres de la provincia de Corrientes que falleció a causa de una atención médica deficiente, después de autolesionarse a modo de protesta por sus condiciones de detención. La presentación no solo tuvo repercusión en los medios de comunicación locales, sino también entre organismos de Derechos Humanos, posicionando de esta manera al OCSoNEA como referencia de información y análisis sobre violaciones a derechos fundamentales.

A consecuencia de lo anterior, desde el área de Derechos Humanos elaboramos notas breves sobre diversos hechos de coyuntura, a partir de información suministrada por organizaciones o referentes sociales. Tal fue el caso de los resultados preliminares, difundidos en 2018, sobre el aumento de desalojos violentos de asentamientos irregulares en Corrientes. Allí se planteó que en un período de seis meses se habían producido en la provincia un total de 7 desalojos que 
afectaron a 175 familias. Del mismo modo, se produjeron notas sobre el aumento de los casos de gatillo fácil en el Chaco y de detenciones arbitrarias en Corrientes.

Durante 2019, hemos participado activamente en múltiples intervenciones, tanto en la provincia del Chaco como en Corrientes. En esas experiencias hemos conocido a otros colectivos organizados, aprendido de sus luchas particulares y hemos estrechado lazos que fueron forjando una red de solidaridad a nivel regional. El contacto y la articulación supusieron la reorganización del trabajo del área, a los fines de conciliar las demandas y tiempos de las organizaciones con las características de las actividades e investigaciones académicas, sin que ello implique un distanciamiento de los objetivos preestablecidos.

Las IV Jornadas Internacionales sobre Conflictos y Problemáticas Sociales/Sextas Jornadas Interdisciplinarias sobre Conflictos y Problemáticas Sociales del Gran Chaco, realizadas el 23 y 24 de mayo de 2019 en la Unne, sirvieron como expresión de los avances alcanzados en cada una de las áreas del OCSO-NEA. Desde el área de Derechos Humanos, participamos exponiendo los resultados finales del informe sobre muertes por el aparato represivo del Estado en la provincia de Corrientes, junto a una comparación aproximativa con los casos de la provincia de Misiones. Nuevamente, la colaboración de la RCDH fue de suma importancia para establecer vínculos, compartir información y elaborar análisis en conjunto con organizaciones de Derechos Humanos de Misiones.

Además, en las mismas jornadas presentamos otra investigación sobre las consecuencias de las detenciones masivas de control policial y los retenes focalizados en la ciudad de Corrientes mediante el registro y sistematización de los operativos de control vehicular -una modalidad específica de acción policial- abarcando el período 10/12/2015-30/04/2019. Esta periodización permite evaluar ciertos contrastes entre dos administraciones, al menos en lo que respecta a la represión preventiva.

La vinculación con organizaciones sociales redundó en la propuesta de coordinación, para les integrantes del área junto con la RCDH, del Taller sobre Violencia Institucional en el marco del Primer Encuentro Provincial de Derechos Humanos, realizado el 3 de agosto en el CCD Ex Regimiento de Infantería № 9. A partir de allí, comenzamos a planificar una serie de visitas a organizaciones del interior de Corrientes para difundir el informe sobre muertes por el aparato represivo del Estado y evaluar las alternativas de articulación con el OCSo-NEA. Pudimos concretar una visita a la localidad de Goya, donde fuimos recibidos por la organización MEDEHS (Memoria, Derechos Humanos y Solidaridad). Además de presentar el informe, surgieron reflexiones sobre casos ocurridos en esa ciudad, la segunda en habitantes de la provincia de 
Corrientes, así como otros no contemplados en el informe (apremios ilegales, persecución y hostigamiento policial).

En agosto de 2019 colaboramos en la organización de la Primera marcha provincial contra el gatillo fácil. Este trabajo, en conjunto con la RCDH, nos permitió conocer a familiares de víctimas de esta modalidad de represión estatal, a las que se reunió para encabezar la movilización. Así, se conocieron historias de casos olvidados, algunos impunes, como el de Pedro Aguirre, Salvador Meza y Ramón Arapí. La marcha fue muy convocante, con participación de organizaciones sociales de base y otros sectores. Los datos más sobresalientes del informe elaborado por el OCSo-NEA fueron incorporados al documento final y leídos durante el acto frente a la Jefatura de Policía.

En el marco del IV Congreso de Filosofía de la Red Norte Grande, llevado a cabo del 25 al 27 de septiembre de 2019 en la Unne, presentamos el Informe sobre muertes por el aparato represivo del Estado en la provincia de Chaco (2008-2018) y nuevos avances en la investigación sobre los operativos de control policial en Corrientes.

\section{Sujetos y subjetividades en la extensión universitaria}

Siguiendo los planteos de Humberto Tommasino (2009), podemos enmarcar nuestra experiencia extensionista por fuera del paradigma clásico -el difusionista- de la extensión universitaria y más bien próximo a los planteos del autor, quien entiende a esta como "un proceso dialógico y crítico, redimensiona a la enseñanza, al aprendizaje y a la investigación" (Tommasino, 2009: 6). En este sentido, desde el área de Derechos Humanos, hemos podido experimentar ese encuentro con el otro y su conocimiento al tomar contacto con una organización no gubernamental como la Red Corrientes de Derechos Humanos.

$\mathrm{El}$ autor de referencia sostiene respecto al conocimiento que hay que "dejar de pensar en relación de partes para pensar en procesos interconectados dialécticamente y que implican participación activa en su construcción" (Tommasino, 2009: 4). Coincidimos con tal premisa y podemos contar desde nuestras experiencias los avatares de la investigación encarada, la cual había partido de las inquietudes del grupo para después ir transformándose en la intersección con la organización. Al comenzar la tarea extensionista, sin haber siquiera tenido una reunión con la ONG, teníamos la percepción de que la misma era una suerte de "fuente de información" de la cual podríamos nutrirnos para desarrollar la investigación. En ese sentido, desde una mirada crítica y retrospectiva, habíamos comenzado fuertemente influenciados por el paradigma clásico extensionista, que no ve en el otro un sujeto de saber, sino un objeto. 
La pedagogía de Freire nos propone una relación de otro tipo, donde el rol del educador sigue siendo importante, pero ya no preponderante en su relación con el educando. De ahí que el educador que se considere revolucionario debe ser consciente de que, al enseñar, está aprendiendo (Freire, 2005). En nuestro caso, se trata de aprender y respetar los saberes obtenidos por las organizaciones sociales a lo largo de su experiencia vivida para construir junto con ellas una comprensión más rigurosa de la realidad.

En este sentido, nos parece pertinente comentar cómo fue nuestro proceso con la Red Corrientes de Derechos Humanos. En una primera instancia, la intención era obtener de la organización civil información que había registrado de manera rudimentaria sobre casos de muerte a manos de agentes policiales y penitenciarios en la provincia de Corrientes. La idea era poder construir una matriz de datos para hacer observable la conflictividad social que estaría operando de manera estructural, hilvanando todos estos hechos que, a simple vista, podrían parecer aislados.

Sin embargo, una vez proporcionada la información, comenzó un proceso sumamente interesante de puesta en diálogo y discusión de los datos sistematizados. Nos encontramos, por ejemplo, con que la organización no se conformó con la normalización y digitalización de sus listados, sino que además quiso -y fue aceptado- participar de la elaboración de la base de datos y el análisis de los casos. Así, nos propusieron el empleo del concepto "muerte por cárcel" para englobar los casos de personas presas fallecidas tanto dentro como fuera de la cárcel. Este término -que proviene de la Comisión Provincial por la Memoria (Buenos Aires), con la que la RCDH está vinculada- nos permitió abordar la problemática específica de estas muertes en el primer informe del área.

La producción de conocimiento científico es también un elemento en disputa permanente entre facciones antagónicas y, por lo tanto, no podría decirse que exista "neutralidad" al investigar la sociedad, sin importar la metodología que se emplee. Al respecto, las producciones que desde el área de Derechos Humanos hemos venido elaborando en conjunto con la Red Corrientes de Derechos Humanos deben ser entendidas como insumos para esta y otras organizaciones en el marco de las luchas que llevan a cabo. Así, por ejemplo, el trabajo sobre operativos de control de vehículos y detenciones arbitrarias por parte de la Policía de Corrientes ha sido incorporado a recientes acciones judiciales que pretenden poner un freno a estos accionares que claramente van en contra de libertades y garantías expresadas e implicadas en la Constitución nacional. Vemos cómo el rol de la extensión puede ser el de contribuir a las luchas, generando instrumentos para acciones concretas, y no solo una apropiación de la información/conocimiento disponible en las organizaciones y sujetos con quienes trabajamos. 
Sin embargo, también debemos considerar críticamente estas producciones teniendo en cuenta que se trata de productos de carácter científico y que han resultado "distantes" respecto al lenguaje, modo de presentación y conocimientos previos, no tanto de la organización con la que se ha trabajado, sino con otres actores o sujetes a quienes podría haber resultado de utilidad (por ejemplo, familiares de víctimas de violencia estatal u organizaciones sociales territoriales que padecen a diario este tipo de violencia).

Estamos convencides de que hay que mantener el diálogo con las organizaciones, ir a su territorio e instalar en la agenda universitaria las demandas y los problemas de estas, pero también pensar y desarrollar un lenguaje comprensible, sin perder su carácter científico. "El tema central es cómo construir herramientas que permitan que amplios sectores de las clases populares puedan acceder a un conocimiento cada vez más distante e incomprensible" (Tomasino, 2009: 8). Quizás allí se esté escondiendo -como un último rescoldo- la influencia del viejo paradigma que en el lenguaje y los gráficos de torta intenta mantener la distancia autoritaria entre educador (creador e intérprete de esos de datos) y educando (mero reproductor del conocimiento "depositado").

\section{Memorias del área de Género y diversidad}

En el área de Género y diversidad del OCSo-NEA hemos trabajado, desde 2017 hasta el momento, como un campo abierto a la construcción de nuevas herramientas y perspectivas teóricas, volcadas al seguimiento de fenómenos conflictivos de la región del NEA. Este componente de apertura implica, en primera instancia, que no existen a priori modelos de trabajo y nociones de conflictividad que estructuren y determinen el tipo de tareas investigativas desarrolladas en el área. La razón de ello reside tanto en el insistente acercamiento que el Observatorio sostiene hacia las organizaciones sociopolíticas afines a las luchas contra la violencia por razones de géneros, como al propio dinamismo de la realidad política que afecta la vida de las poblaciones vulnerables de la región. La proximidad dialógica que el Observatorio ha construido con les actores políticos del feminismo y la diversidad cimenta los criterios estratégicos con los que se reconocen y abordan los fenómenos conflictivos de mayor urgencia.

¿Por qué los feminicidios? Aunque las problemáticas de género y diversidad abrazan un amplio rango de violencias que demandan una tematización objetiva y sostenida, los feminicidios y travesticidios reclaman en nuestro trabajo un lugar destacado, en tanto constituyen no solo la forma más extrema de violencia hacia cualquier ser humano, sino también un fenómeno que parece crecer y reivindicarse casi implacablemente. Aun cuando hablamos de un problema del que podría afirmarse que, sin exagerar, existe en todo el planeta, consideramos 
que cada una de sus manifestaciones guarda siempre un sesgo de singularidad, en la medida en que la violencia en tanto lenguaje se materializa en una saturación de significados con la que se anudan conflictos de racialidad, clase, sexualidad, geografía, etc. De aquí decanta la necesidad y urgencia de contar con un observatorio que, junto a las organizaciones del territorio sensibles al problema, se dedique a registrar y estudiar las características con las que estos tipos de violencias toman lugar en nuestras sociedades.

La primera etapa de registro estuvo facilitada por trabajadores de la comunicación de las provincias del Chaco y Corrientes y algunes miembros también del Observatorio. La información ofrecida consistía en una lista de feminicidios ocurridos en las provincias mencionadas entre 2013 y 2017, documento en el que además se detallan nombres de víctimas y victimarios, edades y lugar de los hechos. Partiendo de estos valiosos antecedentes, fue necesario luego definir y afianzar herramientas y metodologías de trabajo, para extender este registro a todo el NEA y darle continuidad en los años venideros.

Naturalmente, recuperamos la experiencia de otros observatorios y movimientos sociales interesados en la temática. El observatorio Mumala Mujeres, Disidencias, Derechos ${ }^{2}$ contaba ya por entonces con una matriz de registro de feminicidios a nivel nacional, mientras que en el Chaco funcionaba el Observatorio Luisa Bautista ${ }^{3}$ de la Comisión Multisectorial de la Mujer. Estos antecedentes nutrieron y orientaron la labor de nuestro Observatorio, aportando modelos de matrices y variables relevantes o casos no publicados en los medios periodísticos. Sin embargo, lentamente se han podido introducir ciertas modificaciones en la manera en que se nomencla el fenómeno o bien crear otras denominaciones nuevas para hacer aparecer aspectos problemáticos que quedan invisibilizados. Este tipo de transformaciones paulatinas que el trabajo de registro y análisis ha sufrido en su desarrollo se debe tanto al aporte intelectual de las pensadoras feministas como Rita Segato o Marcela Lagarde, por nombrar algunas, como también a las organizaciones territoriales afines a estas problemáticas, con las que se han compartido y discutido resultados, afinando conceptos y estrategias.

La recolección de datos depende casi exclusivamente de los casos que son publicados por los medios periodísticos (salvando pocas excepciones en las que algunas organizaciones brindan cierto tipo de información), esto quiere decir que no es la cantidad real de feminicidios del NEA lo que se plasma en las cifras, sino solo aquella porción de casos que llega a impactar en la prensa periodística de la región. Resulta crucial destacar este detalle no menor, pues este

\footnotetext{
${ }^{2}$ Mumala, organización feminista y política en 22 provincias de Argentina.

${ }^{3}$ El Observatorio de Violencia de Género Luisa Bautista fue creado en 2012 dentro de la Comisión Provincial Multisectorial-Chaco.
} 
margen de error puede hacernos suponer, no sin razones, que quizás una parte de la totalidad de los feminicidios no llega a ser conocida por la población. El Observatorio Luisa Bautista, por ejemplo, ha tendido una red de colaboradores en el interior de la provincia del Chaco, llegando a geografías donde ni la prensa ni el Estado han sabido arribar. Sin embargo, si esto ya resulta una empresa titánica para una sola provincia, es sin duda imposible para organismos que trabajan con vastas regiones geográficas.

Por tales motivos, hemos iniciado un trabajo complementario en el que registramos "muertes de mujeres", apelando al uso de estadísticas vitales de cada provincia. Como toda herramienta metodológica, no carece de problemas, pero como alternativa de registro podrá arrojar un panorama más cercano a la realidad de las mujeres de esta región.

Hasta el momento, hemos sostenido un trabajo de registro de feminicidios en la región del NEA que comprende el período 2013-2020, del que se ha obtenido una media de 26 casos de feminicidios anuales. Los informes de este seguimiento se realizan normalmente dos veces al año; el primero de ellos es publicado entre julio y septiembre, para observar el comportamiento y la velocidad del fenómeno en relación con los años anteriores; el segundo informe cierra el año con el total de cifras registradas y es publicado o difundido por los medios entre diciembre y marzo.

Por su parte, los travesticidios son sumamente escasos en la región, pero demandan un estudio pormenorizado para demostrar algunos condicionantes del seguimiento de este fenómeno. También aquí pueden existir casos omitidos por la prensa o bien la omisión de la identidad de género de la víctima (lo que hace difícil rastrear los casos). Otra de las problemáticas asociadas a esta comunidad es la invisibilización de las violencias sociales que empujan a las compañeras a la muerte. En diálogo con organizaciones LGTBQI+ del Chaco, se ha tenido por necesario incluir la noción de "travesticidio social" para revalorizar las biografías de compañeras que han sido obligadas a vivir en la marginalidad, privadas del acceso a servicios sociales (salud, vivienda, educación, etcétera), a las que se suman casi inevitablemente la prostitución, el consumo problemático de sustancias y la violencia policial, condiciones que cristalizan la expectativa de vida de las mujeres trans y travestis en los 35 años de edad. Se habla de travesticidios sociales porque, aunque tales muertes no tengan un autor con nombre y apellido, como sí ocurriría en un travesticidio normal, existe una deliberada desidia estatal que junto a los discursos odiantes encarnados en el imaginario cultural crean las condiciones para que estas tengan lugar. Desafortunadamente, tampoco se ha podido construir una metodología de seguimiento de travesticidios sociales. No son reconocidas como violencias estatales ni sociales, por lo que no salen en los medios. Tampoco es posible acceder al registro de 
defunciones de los hospitales y por si fuera poco, las estadísticas vitales no reconocen identidades por fuera del binarismo hombre-mujer. El país requiere con urgencia de una modificación en las categorías de sus estadísticas vitales, una epidemiología con perspectiva de género para restituir a la comunidad trans un poco de su memoria o bien para evitar que esta continúe siendo un privilegio CIS.

Los resultados de todos estos procesos y discusiones han sido compartidos y viralizados en numerosas oportunidades por los medios periodísticos de la región, quienes periódicamente consultan las cifras y trabajos de nuestro Observatorio. Además, hemos organizado reuniones científicas en distintas universidades y conversatorios con agrupaciones políticas y territoriales afines a la temática. A continuación, nombramos algunos de estos antecedentes.

En 2017 realizamos una charla con organizaciones feministas de la provincia de Corrientes, en la Facultad de Humanidades, sede Sargento Cabral, Corrientes. Aquí tuvimos la oportunidad de dar a conocer el equipo de trabajo a referentes feministas y presentar algunos avances en el registro de feminicidios. Ese mismo año también participamos de una charla con organizaciones feministas e interesades en el Centro Cultural Alternativo de la provincia del Chaco. En esta oportunidad, se pudo presentar el trabajo realizado ante referentes del movimiento feminista de la provincia.

En 2018 realizamos un "Análisis comparativo entre los femicidios de Chaco y Corrientes entre el período 2013-2018", el cual fue expuesto en las Jornadas científico-tecnológicas de la Universidad de Misiones y también en las Primeras jornadas internacionales de estudios de género del Nordeste Argentino y países limítrofes, y posteriormente publicado en sus actas ${ }^{4}$. Durante ese ciclo también participamos y desarrollamos el informe mencionado en el Seminariotaller: Observatorios Socio-territoriales de América Latina: avances y desafíos, realizado el 30 y 31 de octubre en la Facultad de Ciencias Sociales (UBA), Ciudad Autónoma de Buenos Aires, Argentina. Aquí tuvimos la oportunidad de conocer otros observatorios de la Argentina y países limítrofes.

Como indicamos anteriormente, en el intercambio con las distintas organizaciones y estudios sobre feminicidios fuimos construyendo la perspectiva teórica, tal es así que en las XVI Jornadas de Estudiantes de Filosofía/ I Jornadas Interclaustros del Departamento de Filosofía/ IV Coloquio de Doctorandos en Filosofía y Ciencias Sociales, realizados el 27 y 28 de noviembre de 2018 en la Facultad de Humanidades de la Universidad Nacional del Nordeste, Resistencia, Chaco,

\footnotetext{
${ }^{4}$ Véase Mandinola, Myrian et al. (2018). Actas (1 $1^{\text {a }}$ ed. compendiada) (pp. 19-23). Corrientes: Universidad Nacional del Nordeste, Facultad de Humanidades.
} 
presentamos un "Análisis comparativo entre los feminicidios de Chaco, Corrientes y Misiones en el período 2013-2018, a partir de los aportes de Rita Segato".

En 2019 participamos del taller de Violencia institucional del Primer Encuentro Provincial de Derechos Humanos, realizado el 3 de agosto en el Ex Regimiento de Infantería No 9 (centro clandestino de detención y tortura durante la dictadura) y en la Charla "Yo he tocado a un pobre: neoliberalismo y odio en la Argentina amarilla", en el Centro Cultural Popular (Cecupo), para reflexionar sobre la violencia social y los discursos de odio que encarna el imaginario cultural. Asimismo, presentamos la ponencia "Feminicidios en el Nea Argentino: reflexiones en torno a las particularidades de los casos de la región", en el marco del IV Congreso de Filosofía de la Red Norte Grande, realizado del 25 al 27 de septiembre de 2019 en la Unne.

\section{Memorias del área Ambiente y territorio}

Desde 2018, en el área Ambiente y territorio llevamos adelante un trabajo colaborativo con la organización no gubernamental Red de Salud Popular Dr. Ramón Carrillo ${ }^{5}$. Este trabajo surge como producto de vinculaciones previas: encuentros en las Jornadas sobre conflictos y problemáticas sociales en la región del Gran Chaco (2011) y posteriormente la realización del proyecto de extensión denominado "Desarrollo de material didáctico para la protección de los derechos de poblaciones afectadas por la fumigación con agrotóxicos" (programa La Universidad en el Medio, convocatoria 2015). Nuestra tarea consistió principalmente en relevar y sistematizar denuncias por fumigaciones con agrotóxicos en localidades de la provincia del Chaco, entendiendo que las mismas son formas de expresión de conflictos ambientales relevantes en nuestra región.

Entendemos que los conflictos ambientales no solo son problemas concernientes al ambiente, en tanto síntoma de una serie de cambios en la naturaleza a consecuencia de la acción humana sobre esta, sino que además lo integran una disputa o antagonismo de intereses por el acceso, apropiación, gestión, determinación y uso de los bienes comunes y del espacio (Merlinsky et al., 2018). Esta dimensión espacial no solo se refiere a una región geográfica, sino al territorio entendido como el ámbito en el cual se configuran las condiciones de existencia, se dan expropiaciones y apropiaciones que enmarcan a las distintas luchas que protagonizan las

\footnotetext{
${ }^{5}$ La Red de Salud Popular Dr. Ramón Carrillo es una entidad que nuclea a un amplio grupo de personas no profesionales y profesionales como abogades, médicos/as, ingenieres agrónomes y personal técnico que trabajan desde hace más de una década asesorando a pobladores, tanto urbanos como rurales, afectados por la fumigación con agrotóxicos en distintas localidades de la provincia del Chaco, acompañando denuncias, realizando propuestas de producción sin agrotóxicos, promoviendo acciones de atención primaria en salud y atención primaria ambiental, lo cual les permite crear lazos en toda la extensión del territorio chaqueño.
} 
diferentes fracciones y sujetos sociales y se despliegan relaciones de poder (Rozé, 2007). Desde este marco, el registro y análisis de las acciones de denuncia permitirían generar un mapa de la conflictividad ambiental concerniente a las fumigaciones con agrotóxicos, así como analizar sus características e indagar en sus causas estructurales.

En un primer momento, tomamos la decisión de sistematizar por completo el archivo de la Red, teniendo en cuenta lo que la ONG había propuesto y lo que creíamos que podíamos llegar a hacer: generar una herramienta que les sea de utilidad para ordenar la información concerniente al desarrollo de los conflictos y que, a su vez, dicha herramienta nos permita realizar un análisis que logre contribuir a la visibilización de los mismos.

En retrospectiva, podemos notar que la noción de la tarea que pensábamos desarrollar era la de un servicio, algo que Freire (1984: 21) tematiza cuando se refiere a las connotaciones semánticas que el término extensión implica: como obra de un sujeto activo que extiende un contenido a una otredad que lo recibe. Aparece nuevamente aquí la extensión transferencista/difusionista, sustentada en la idea de la Universidad como propietaria de un saber, técnica, ciencia o tecnología pasible de ser transmitida, depositada y/o entregada a algo externo a ella (Tommasino y Cano, 2016). Por lo tanto, nos parece interesante para el presente escrito señalar y problematizar cómo esto que inició con una idea esquemática y mecánica en torno a trabajos con archivos luego significó trazar nuevas líneas por fuera del recorrido que habíamos preestablecido.

Empezamos el trabajo yendo semanalmente a la sede donde funciona la ONG para leer los documentos que tienen disponibles en sus archivos, siempre acompañadas por algún miembro de la organización. Como primera tarea, elaboramos una matriz para relevar los datos obtenidos de tales documentos para, en principio, contabilizar las denuncias por fumigaciones con agrotóxicos que había por localidad en toda la provincia, teniendo en cuenta que la muestra se limitaba solo a los archivos mencionados. Esa tarea se nos volvió compleja por varias razones que explican el modo de trabajo que fuimos elaborando y las relaciones humanas que se fueron creando a partir de ello, como también nos permiten identificar problemáticas propias que se insertan en la vinculación entre la academia y la sociedad.

En primer lugar, al provenir del campo disciplinar de la Filosofía, el lenguaje jurídico específico de los documentos nos era sumamente ajeno, con lo cual debíamos comenzar por realizar un proceso de inmersión en esas categorías. Podemos identificar cómo la rotación de roles, según la denominación de Tommasino (2009), se hacía presente al ir consultando y preguntando a les integrantes de la Red, quienes por el hecho mismo de ser especialistas en el tema y además militantes comprometides en las causas, comprendían mejor los datos que allí se 
expresaban. Este proceso de diálogo nos llevó también a conocer los detalles y pormenores de las acciones de denuncia. En este sentido, integrantes de la ONG nos comunicaron la importancia de la lectura de los documentos de sus archivos por parte de personas externas para reconstruir los procesos atravesados y recuperar información relevante según nuevos puntos de vista.

La segunda razón consiste en que, una vez establecidos los criterios de cómo y qué datos relevar, notamos que las denuncias por fumigaciones en sí mismas eran minoritarias en comparación con la gran cantidad de reclamos relacionados a esos hechos. Además, los casos conflictivos abarcaban una complejidad que se expresaba en el devenir de esos documentos y que excedían a la fumigación en sí. Los expedientes permitían trazar una cronología de los conflictos y comprenderlos en su génesis. Por estos motivos, decidimos ampliar la delimitación de las acciones de denuncia que iban a relevarse y al mismo tiempo recortar la amplitud temporal y espacial del registro debido a la gran extensión del archivo. Esto, a su vez, permitió una mayor profundización en casos específicos.

En 2018 expusimos sobre los primeros avances de este relevamiento y sistematización de datos respecto al caso del conflicto por fumigaciones con agrotóxicos en La Leonesa y Las Palmas, en las XVI Jornadas de Estudiantes de Filosofía y Primeras Jornadas Interclaustros del Departamento de Filosofía, en la Unne.

En este punto, algo que en principio parecía ser automático, se nos mostró como un proceso de diálogos y decisiones conjuntas en constante construcción con la Red, a la vez que se entretejían vínculos humanos muy valiosos. Notamos que era necesario además trazar una continuidad hasta el presente para integrar también las alternativas que les actores denunciantes proponían ante los conflictos atravesados. Esto último fue explícitamente sugerido por personas de la organización, y en ese contexto presentamos un proyecto de extensión en el marco de la convocatoria UNNE en el Medio para ser ejecutado durante 2019, con el objeto de hacer ese trabajo con una mínima financiación. A su vez, dicho proyecto se convertía en una oportunidad y una invitación: la de introducirnos al territorio, con la posibilidad de conocer a las personas que protagonizaron los conflictos que estudiamos. En paralelo, ampliando el período temporal de las acciones de denuncia y revisando algunas definiciones para el análisis del conflicto de La Leonesa y Las Palmas, expusimos en las Cuartas Jornadas Internacionales sobre Conflictos y Problemáticas Sociales/ Sextas Jornadas Interdisciplinarias sobre conflictos y Problemáticas Sociales del Gran Chaco de la Unne y en el IV Congreso de Filosofía de la Red Norte Grande en la Unne. 
Una vez aprobado el proyecto al que denominamos ¿Cómo enfrentar a los agrotóxicos? Sistematización de modos de acción desde comunidades afectadas por fumigaciones en la Provincia del Chaco, en el período 2010-2015, continuamos con la tarea de relevamiento y sistematización de las denuncias (ahora también digitalizando el archivo de la Red). Pero ya solo respecto a tres localidades determinadas, con el período de tiempo ampliado según el inicio y finalización de cada caso. Esas localidades fueron: Colonias Unidas, La Leonesa/Las Palmas y Pampa del Indio. En principio, elaboramos tres informes (uno por cada selección), los cuales presentamos en su localidad correspondiente, para compartir la información que habíamos elaborado y para que la misma pudiera posteriormente ser rectificada, discutida y ampliada conforme a la participación de les protagonistas y sus comunidades. De allí surgieron encuentros y entrevistas semiestructuradas con les actores mencionados, lo cual debemos señalar, nos ha dejado importantes aprendizajes y reflexiones sobre nuestras prácticas.

Producto de esos encuentros, revisamos nuestras estrategias de comunicación. Comprendimos la importancia del diseño de la información expresada en los informes. En principio, vimos que era necesario hacerlos más accesibles a les lectores en general (e incluso más didáctico para las instancias de intercambio), algo que con el aporte de nuevas compañeras que integraron el proyecto y que, por ejemplo, contaban con conocimiento de lengua Qom, pudo empezar a trabajarse. Todavía es una tarea pendiente mejorar estos aspectos. Además, durante 2019, en el marco del desarrollo de nuestro proyecto de extensión, realizamos presentaciones y jornadas de capacitación para el relevamiento de datos de los casos mencionados.

Fue así como a partir de las instancias en las que el acceso al territorio fue posible, una pregunta que para nosotras ya tenía una respuesta predeterminada, instaba a ser revisada. El trabajo del área y por ende del Observatorio, el cual se enmarca en el ámbito universitario: ¿Se limitaba solamente a generar información de utilidad para las organizaciones con las que trabajamos? ¿Qué rol le estábamos atribuyendo a aquelles actores que llevaron adelante las acciones de denuncia y reclamo que nosotras pasamos leyendo cada semana? El contacto con elles, ¿sólo contribuiría a la rectificación de dicha información o al agregado de otras nuevas? 0 más bien, ¿podríamos generar oportunidades para que la sociedad logre interpelar el trabajo que lleva adelante la Universidad?

Estas y muchas otras preguntas fueron surgiendo a medida que teníamos la oportunidad de dimensionar que los casos con los que trabajamos implican de alguna manera introducirnos en las historias de lucha que diversas familias han ido construyendo. En comunidades que a su vez han padecido los efectos de una problemática ambiental y social estructuralmente arraigada en 
sus territorios, a raíz de la imposición del agronegocio, con todo lo que eso implica: el deterioro de la salud, la imposibilidad de un ambiente sano, las dificultades para continuar con el desarrollo de sus actividades productivas y de sustento, el acaparamiento de los bienes comunes, entre otras consecuencias. Lo que representa la permanente vulneración de sus derechos y ante lo cual construyen resistencias.

\section{Algunas vinculaciones en el territorio}

En La Leonesa y Las Palmas, primeras localidades a las que asistimos con el objeto de compartir el trabajo que veníamos realizando, la puesta en común del informe no se constituyó como el acto central de la jornada. Aquella instancia se convirtió más bien en una oportunidad para el reencuentro entre les actores del territorio y les integrantes de la Red, para reflexionar sobre el proceso de lucha que habían forjado hace casi una década y para ponerse al tanto de qué características había adoptado este proceso en la actualidad. Para idear estrategias en conjunto $y$, sobre todo, para continuar en alerta ante un modelo productivo nocivo.

En el caso del conflicto en Colonias Unidas, la presentación del informe se dio durante noviembre de 2019 en la EFA Arraigo Campesino, teniendo como destinataries a alumnes del Nivel Secundario y estando en presencia de les actores que habían formado parte de las acciones sistematizadas. El momento se conformó como la posibilidad de revalorizar un proceso de lucha desconocido por muches de les estudiantes, el cual había obtenido como resultado más favorable, el cese de las fumigaciones en aquel territorio. Luego de la presentación, los gráficos varios que habíamos obtenido a partir del relevamiento y la sistematización fueron adquiriendo nuevos sentidos. A través de una entrevista grupal semiestructurada que habíamos programado, les actores en primera persona relataban cómo habían transitado el conflicto y cómo las acciones colectivas les habían resultado efectivas para la defensa de sus modos de vivir y habitar el territorio. Enfatizaron el valor de rememorar dicho proceso, instando a que otras comunidades que estuvieran atravesando por condiciones similares puedan conocer su causa.

Por último, al presentar los resultados del informe correspondiente al caso de Pampa del Indio durante diciembre de 2019 en la EGB Nº 522 Rubén Saravia, de Campo Medina, estuvieron presentes, además de les actores involucrades, funcionaries públicos del actual gobierno del Chaco: la subsecretaria de Ambiente y Biodiversidad, acompañada por funcionarios de esa dependencia, quienes visualizaron lo que el informe indicaba: que durante el período 2010-2018, la mayoría de las denuncias por fumigaciones y sus consecuencias estaban dirigidas a organismos del Estado de manera reiterada, lo cual permitía dar cuenta de la responsabilidad estatal en medio del proceso. Algo que personas de la Red de Salud han considerado como un 
momento en el que pudieron dimensionar el alcance del ordenamiento y sistematización de las acciones de denuncia, con su divulgación en esos informes.

En 2019, además, habíamos Ilevado a cabo otras actividades tales como conversatorios con la organización Somos Monte en el contexto de la Huelga mundial por el clima, sumándonos a las movilizaciones y marchas; acompañamos en la presentación de la línea de indumentaria NO'OXONEC de algodón agroecológico producido en Pampa del Indio por las mismas comunidades en lucha contra las fumigaciones y la Red de Salud, entre otras.

Sin embargo, consideramos que las actividades más significativas para el presente escrito son aquellas que nos llevaron a re-pensar nuestros haceres conjuntos. A identificar cómo, a pesar de nuestra inexperiencia, logramos acercarnos a prácticas más integrales, las cuales implican necesariamente un tipo particular de articulación entre los procesos de aprendizaje y enseñanza, investigación y extensión. A su vez, generar un espacio donde es posible que dichos procesos de enseñanza y aprendizaje se den por fuera del aula, y puedan generarse y operar en terreno, junto a la gente, partiendo de los problemas que la gente tiene, intentando junto con ella encontrar alternativas. Esta situación puede reconfigurar el acto educativo y fundamentalmente redimensionar el poder que circula en les diferentes actores del proceso (Tomasino, 2009).

\section{Transformaciones y desafíos desde la experiencia}

Es notable que en los distintos procesos de trabajo colaborativo se produjeron cambios significativos en los imaginarios, objetivos y expectativas que se dieron conforme a nuestro encuentro con las organizaciones con quienes trabajamos. Confiamos en las personas que las integran como conocedoras de las temáticas que buscamos sistematizar y conocer. En este sentido, el concepto de diálogo de saberes que plantea Castro Gómez (2007) nos parece acertado para mirar los procesos que fuimos llevando a cabo y los momentos de toma de conciencia que ante determinadas situaciones nos han interpelado, ya que ese diálogo es entendido como "la posibilidad de que diferentes formas culturales de conocimiento puedan convivir en el mismo espacio universitario" (Castro Gómez, 2007: 87).

Ese gran desafío implica salirnos del ideal de objetividad y asumirnos como parte de un entramado social y cultural históricamente formado. Parte de eso es identificar en nuestras prácticas los mismos supuestos que pretendemos no reproducir. Por ejemplo, buscando mantener cierta actitud crítica ante el uso de metodologías llamadas cuantitativas o cualitativas, que se definen unilateralmente y que tienen en mayor o menor medida pretensiones de objetividad o de observar sin ser observades (Castro Gómez, 2007). Nuestro intento fue 
adaptarlas con el mayor cuidado posible, teniendo en cuenta las alternativas más apropiadas para nuestras condiciones y a pesar de todas las limitaciones con las que contábamos.

Integrar las voces de les actores coparticipantes juega en esa encrucijada: dar lugar a la palabra del otre y a la vez buscar abarcar de manera estructural las condiciones de posibilidad de esas palabras, además de generar un vínculo y un compromiso que entendemos es necesario preservar y respetar. Pensamos que nuestra tarea se enmarca en esa dicotomía entre lo particular y lo general que tiene que ver con una lógica de tiempos académicos y burocráticos que nos condicionan. Abordar el trabajo con organizaciones es establecer vínculos dialógicos que prioricen lo humano y la escucha. Esto da lugar a que los tiempos de trabajo se vean modificados por la vinculación con las organizaciones que, por lo general, se mantienen en un proceso constante de acción política que motoriza los contenidos de las investigaciones. Entonces, para realizar un trabajo comprometido, profundo y con respeto por las culturas intervinientes, el espacio y reconocimiento en lo académico debe ser integral como lo plantean Tommasino y Cano (2016), algo que en las condiciones actuales presenta problemas por la dificultad de conciliar esos tiempos y la falta de recursos humanos para llevar a cabo las actividades. Sin embargo, un aporte sustancial que integrantes de la Red de Salud nos han compartido es la valorización de nuestra vinculación como Observatorio a la Universidad pública y gratuita para el trabajo conjunto, entendiendo que esta institución es un marco que permite que voluntades colectivas e individuales se encuentren y construyan conocimiento, métodos y estrategias compartidas.

Creemos en el valor de la investigación canalizada por el trabajo extensionista que permite a docentes, estudiantes e investigadores poner los conocimientos "provisorios y relativos" al servicio de la comunidad para estimular el desarrollo de alternativas y salidas a los conflictos sociales (Bordoli, 2006). Consideramos de igual manera que estos esfuerzos no son animados por formas de asistencialismo, porque tal como explica Bordoli al hablar del trabajo extensionista, la Universidad no es un agente promotor de bienestar en sí misma, sino de cultura y saber, lo que de forma indirecta tiende a producir las condiciones de posibilidad para mejorar la calidad de vida de las personas. Por estas razones, es que como colectivo trabajamos para poner al alcance de las organizaciones sociales información clara, relevante y necesaria para el abordaje de las conflictividades específicas que nos atraviesan como sociedad (Bordoli, 2010). Por esta misma razón, confiamos en la potencialidad del trabajo extensionista y su acompañamiento no paternalista, puesto que no se trata de llevar un conocimiento a quienes no lo poseen, sino de producirlo creando mecanismo de comunicación e integración dentro y fuera de la Universidad (Ortiz-Riaga y Morales-Rubiano, 2011). 
Es necesario que el trabajo extensionista evite reproducir modelos empresariales donde la producción de saberes y la atención de las necesidades comunitarias solo representan una posibilidad de lucro (Serna, 2017). Extender desde la Universidad debe implicar algo más que la apertura de sus puertas o la inclusión de nuevos sujetos en su comunidad. El trabajo extensionista de la Universidad necesita albergar su propia transformación, derribando prejuicios en la enseñanza, la tecnocracia, la investigación o la experticia, siendo capaz de hallar en el espacio alo-institucional sujetos de conocimientos capaces de colaborar e incorporar importantes herramientas de trabajo y reflexión a la tarea que la extensión asume. Por lo que es necesario pensar una investigación participativa que apele al conocimiento popular que se genera en los espacios de resistencia como son las organizaciones con las que trabajamos. Y a su vez realizar el trabajo de transformarlo en saberes sociales que queden registrados y puedan ser accesibles a la comunidad y no se vuelva solo un paper en una jornada.

En consecuencia, el modelo de extensión que más nos interesa desempeñar y ver en nuestro recorrido es un modelo crítico que busque esos diálogos de saberes. Aun así, identificamos que hay algunos elementos anclados en concepciones transferencistas en nuestro hacer y que son necesarias revisar y hacer explícitas justamente para lograr ese objetivo. Siempre está el riesgo de reproducir la voz de la dominación, como lo plantea Rebelatto (2000: 71), en cuanto a los desafíos de la investigación: "Una tarea clave del intelectual orgánico en la investigación participativa sea enfrentarse y ayudar a que los grupos se enfrenten con la contradicción, mostrar las incongruencias (obviamente también sus propias incoherencias), descubrir potencialidades como también señalar extrañamientos. Debemos ser investigadores de la esperanza, no de la resignación. Investigadores desafiantes, no meros facilitadores". Por esto, entendemos que la relación entre lo académico y lo no académico debe ser constantemente problematizada.

\section{Conclusión}

Desde las memorias descritas, hemos pretendido dar a conocer las estrategias y acciones que están detrás de nuestra metodología de investigación académica, entendiendo que es en ese proceso en el cual se construye el conocimiento junto con y para las organizaciones de la sociedad civil. Cada área mantiene su particularidad de acuerdo con la temática que aborda y con las relaciones consolidadas con las organizaciones. Por eso mismo, pudimos ver cómo las preguntas y reflexiones de cada una surgen desde experiencias concretas diversas, pero son analizadas desde una perspectiva común. 
Como equipo, hemos atravesado varios desafíos a lo largo de estos años. En primer lugar, el marco estrictamente universitario otorga un alto grado de incertidumbre por la renovación anual de los proyectos de extensión y la necesidad de destinar gran parte de los esfuerzos a preparar los proyectos de la etapa siguiente. En segundo lugar, la cambiante situación de disponibilidad temporal de estudiantes y egresades, así como la escasa valoración que tienen las actividades de extensión para currículums, dedicaciones y concursos dificulta a veces la capacidad técnica para el registro y análisis de todos los temas en cada área. El desafío que atravesamos actualmente es la ampliación del equipo.

Para sortear estos obstáculos, el Observatorio comenzó a tejer alianzas con investigadores y observatorios de otras provincias, a fin de coordinar acciones y ampliar las posibilidades de registro. Por otro lado, se realizan frecuentemente charlas, talleres y seminarios para les integrantes como también para otras organizaciones que deseen comenzar a sistematizar sus registros.

Asimismo, más allá del análisis y procesamiento de datos, la elección de qué observar, sistematizar y analizar, qué datos son relevantes y cuáles no para las organizaciones sociales en sus luchas, es una decisión que tomamos en conjunto, que se basa en convicciones y voluntades individuales y compartidas. En consecuencia, se ha consolidado un equipo de trabajo que se especializa continuamente en su área.

Finalmente, a lo largo de estas páginas pudimos identificar cómo el ámbito no académico nos ha enseñado a pensar otras formas de construir conocimiento, a preguntarnos el para qué hacerlo, además de hacer evidente la contraposición entre los tiempos y ritmos a la hora de hacer este tipo de trabajos colaborativos. Consideramos que las actividades de extensión tienen ese desafío y esa riqueza: la de estar en los bordes entre la academia y todo lo que ella pone por fuera, intentando generar cambios sociales, siendo partícipe de ellos, mientras se convive con dinámicas que muchas veces dificultan ese mismo devenir.

\section{Bibliografía}

Bordoli, E. (2006). Aportes para la conceptualización de los Programas Integrales en la UdelaR [CD]. Montevideo: CSEAM. (2010). Aportes para pensar la extensión universitaria. Extensión en obra, 13.

Castro Gómez, S. (2007). Decolonizar la universidad: La hybris del punto cero y el diálogo de saberes. En Castro Gómez, S. y Grosfoguel, R. (eds.) El giro decolonial: Reflexiones para una diversidad epistémica más allá del capitalismo global (pp. 79-91). Bogotá: Siglo del Hombre Editores. 
Freire, P. (1984). Extensión o Comunicación: La concientización en el medio rural. Uruguay: Ediciones Siglo XXI. (2005). Pedagogía del oprimido. México: Ediciones Siglo XXI.

Merlinsky, G.; Toledo López, V.; Schmidt, M.; Fernández Bouzo, S.; Tobías, M.; Langbehn, L. y Capalbo, T. et al. (2018). Defender lo común: qué podemos aprender de los conflictos ambientales. Ciudad Autónoma de Buenos Aires: Universidad de Buenos Aires, Instituto de Investigaciones Gino Germani.

Ortiz-Riaga, M.C. y Morales-Rubiano, M.E. (2011). La extensión universitaria en América Latina: concepciones y tendencias. Educación y educadores, 14(2), 349-366.

Pratesi, A.; Graciosi, M. y Román, M. (2018). Observatorio de conflictos sociales del Nordeste Argentino. Abordaje conceptual y metodológico. De Prácticas y discursos. 7(9), 117-140. Resistencia: Universidad Nacional del Nordeste, Centro de Estudios Sociales.

Rebelatto, J.L. (2000). Ética de la Liberación. Montevideo: Nordan Comunidad.

Serna Alcántara, G.A. (2007). Misión social y modelos de extensión universitaria: del entusiasmo al desdén. Revista Iberoamericana de Educación, (43)3.

Tommasino, H. (2009). Generalización de las Prácticas integrales. Los aportes de la extensión a su implementación. Uruguay: Facultad de Psicología, Universidad de la República del Uruguay. Recuperado de: https://psico.edu.uy/sites/default/files/tommasino_practicas_integrales_0.pdf

Tommasino, H. y Cano, A. (2016). Modelos de extensión universitaria en las universidades latinoamericanas en el siglo XXI: tendencias y controversias. Universidades, (67), 7-24. Recuperado de: $h$ ttps://www.redalyc.org/articulo.oa?id=373/37344015003

Rozé, J. (2007). La lucha de clases, hegemonía, territorios e identidades en Chaco del siglo XXI. En Lucha de clases en el Chaco contemporáneo (pp. 403-452). Resistencia: Librería de la Paz. 